\title{
Dynamic Conditional Dependence for Turkey Earthquake Data: CD Vine Copula Approach
}

\author{
Ayşe METİN KARAKAŞa,* iD, Aslıhan DEMİR ${ }^{\mathrm{b}}$ iD, Sinan ÇALIK ${ }^{\mathrm{b}}$ \\ a Department of Statistic, Faculty of Art and Sciences, Bitlis Eren University, Bitlis, Turkey \\ b,Department of Statistic, Faculty of Sciences, Firat University, Elazı $\breve{g}$, Turkey
}

\section{ARTICLE INFO}

\section{Article history:}

Received 14 October 2021

Received in revised form 16 November 2021

Accepted 16 November 2021

\section{Keywords:}

Dynamic Conditional Dependence,

Copula,

CD Vine Copula

\begin{abstract}
A B S T R AC T
The objective of this research was to use Turkey's major fault zones, which are located on fault lines, to describe the dependency structure. The current study also intended to show the dynamic structure of the conditional dependencies of the earthquake data in Turkey in terms of depth and magnitude, using the CD-vine copula method. Conditional dependence, also known as the CD-vine method, makes obtaining a complex dependency structure simpler. The current research uses 30 years of data from the Eastern Anatolian Fault line and the North Anatolian Fault line to describe the dynamic conditional dependency structure. As a consequence, this dependency structure is represented graphically and numerically in tables and graphs.
\end{abstract}

(C) 2021. Turkish Journal Park Academic. All rights reserved.

\section{Introduction}

[1] Investigated the dynamics co movement between capital markets in ASEAN Exchanges for during period of 2012-2013 and studied based on C-D Vine copula approach found the dynamics co-movement among of capital markets in ASEAN Exchanges. [2] stuided focuses on the pattern of relation among major world exchanges such as the United States stock market (S \& P 500), European stock markets (the united kingdom and German), Asia stock markets (Japan and China) and ASEAN stock markets (Indonesia and Philippine) in the pre-crisis period $(2000 \sim 2008)$ and post-crisis period (2000 $\sim 2016$ ). Using the C-D Vine copula approach and in their results, they found the dynamic change between stock markets in the world financial markets. [3] used the vine copula analysis to analyze the composite stock price index and some of the macroeconomics (inflation, IDR to USD exchange rate and interest rate). In addition, the dependent structure was applied to obtain the common density function and they calculated the expected value as a copula regression model. [4] presented, the canonical vine (C-vine) and D-vine copulas provide functions and tools for the extraction of stolen Rpackage presents CDVine. [5] examined the daily informational structure and common movements of the Thai Baht (THB) and Malaysian Ringgit (MYR) currencies in the period 2006-2013. [6] proposed vine copula mixes for the dependency structure hidden in multidimensional data. They tested a finite number of $\mathrm{C}$ - and D-vine model mixtures using the same copula family and finally studied the original CD-vine model, which enables the analysis of the dependency structure between many variables. [7] examined the relationship between bitcoin and other cryptocurrency indicators using the CD Vine Approach method.[8] To uncover and fully understand complex and latent dependence patterns in multivariate data, they proposed a D-vine copula mix that incorporates D-vine copula into a finite mix model. In addition, the model they propose can facilitate the scope of their multivariable complex and confidential content models.[9] presented the design of the $\mathrm{R}$ package copula, other implementation details.[10] have presented an intuitive systemic risk model for analyzing complex complex problems between different borrowers.

\footnotetext{
* Corresponding author. Tel.: +904342220020

E-mail address: aysekarakas5767@ gmail.com

ORCID : 0000-0003-3552-0105 (A. Metin Karakaş), 0000-0003-4532-1564 (A. Demir) , 0000-0002-4258-1662 (S. Çalık)
} 
Then, they used the state-of-the-art canonical (C-) and D-vine bridge to investigate the correlation structure between the divisions between the groups. [11] have used vine copula models to capture climate-efficiency dependency patterns, including the occurrence of extreme events (i.e. tail dependencies). Inc et al. [12] obtained approximate solutions of nonlinear time-dependent generalized Fitzhugh-Nagumo equation with time-dependent coefficients and SharmaTasso-Olver equation subjected to certain initial conditions and showed that this method is efficient and convenient; thus it can be applied to variety of problems. The approximate solutions are compared with the exact solutions. Then, Acay and Inc [13] proposed non-local singular fractional operators and examined this model, which has a very important place in everyday life. In 2020, Houwe et al. [14] studied analytical solutions of nonlinear differential equations (DED) with fractional derivatives and used the discrete tanh method for the calculations. In addition, Akinlar et al. [15] take an epidemic system with additional fractional white noise, build a new SIRS model and mix it into the fractional model, to show that epidemics can be modeled more competently in fractionalstochastic environments than those modeled by fractionalstochastic environments. deterministic differential equations. stochastic systems and their chaotic behavior at unscathed and endemic equilibrium points have been studied. Later, Akınlar et al. [16], optimal control formulations, digital solutions, stability analysis for the fractional malchus model is considered a new contribution because it is studied for the first time in this article. Later in the same year, Korpinar et al. [17] analyzed the fractional-cubic-cubic-stochastic non-linearstochastic schrödinger equation, which describes the propagation of the solitons through the optical fibers, and used it to obtain stochastic solutions in space White noise with hermit transform. In addition, Hashemi et al. [18] used the diagram of Adams-Bashforth-Moulton (ABMS) to determine the approximate solution of the fractional three-dimensional chaotic process at varying degrees, showing the results of the simulation. In this study, it is a question of explaining the dependency structure using the Copulule CD-Vigne approach for the main zones of faults located on the fault lines of Turkey. Conditional dependency, in other words CD-vine approach, provides convenience in obtaining the complex dependency structure. The present study explains the dynamic conditional dependency structure using 30 years of data of the regions on the Eastern Anatolian Fault line and the North Anatolian Fault line. As a result, this dependency structure is presented in graphics and tables.

\section{Materials and Methods}

Definition 2. 1. 1.Copula Function

A copula is a multivariate distribution whose marginals are all uniform over $[0,1]$. For a $n$-dimensional vector $U$ on the unit hyper cube, a copula $C$ is defined as,

$C\left(u_{1}, u_{2}, \ldots, u_{n}\right)=\operatorname{Pr}\left(U_{1} \leq u_{1}, U_{2} \leq u_{2}, \ldots, U_{n} \leq u_{n}\right)$
This definition is the main result of the Sklar's theorem [17], namely building block of the theory of copulas, given below. Theorem 2.1.2. (Sklar's Theorem) Let $F$ be a $n$-dimensional distribution function with univariate margins $F_{1}, F_{2}, \ldots, F_{n}$. Let $A_{i}$ define the range of $F_{i}$ and $A_{i}=[-\infty, \infty]$ where $i=1,2, \ldots, n$.At that case, there exists a copula function $\mathrm{C}$ whole $\left(x_{1}, x_{2}, \ldots, x_{n}\right) \in[-\infty, \infty]$

$F\left(x_{1}, x_{2}, \ldots, x_{n}\right)=C\left(F\left(x_{1}\right), F\left(x_{2}\right), \ldots, F\left(x_{n}\right)\right)$

where the random variables $\left(X_{1}, X_{2}, \ldots, X_{n}\right)$ are assumed to be continuous.

Definition 2. 1. 2. Kendall Tau and Spearman Rho rank correlations are defined as Spearman $\rho$ and Kendall $\tau$ For both rank based correlation measures, the general term in their formulations is the rank of the observation, indicated below

$$
\begin{aligned}
& \widehat{\rho}_{S}(X, Y)=12 \iint_{U}(C(u, v)-u v) d u d v \\
& \widehat{\rho}_{\tau}(X, Y)=4 \iint_{U} C(u, v) \partial C(u v)-1
\end{aligned}
$$

where, $U=I^{2}$ is the unique square.

\section{Definition 2. 1. 3.Tail Dependence}

The case of queue dependency is directly related to the extreme value relation defined mainly as a function of the queues. Suppose $X$ and $Y$ are two random variables having distributions $F_{X}$ and $F_{Y}$ respectively. Therefore, two important asymptotic measures for tail dependence, called upper and lower tail dependency coefficients, are mentioned below.

$$
\begin{aligned}
& \lambda_{l}=\lim _{u \rightarrow 0} P\left(\mathrm{~F}_{x}(x) \leq u \mid F_{y}(x) \leq u\right)=\lim _{u \rightarrow 0} C(u, u) / u \\
& \lambda_{u}=\lim _{u \rightarrow 1} P\left(\mathrm{~F}_{x}(x)>\mathrm{u} \mid F_{y}(x)>u\right)=\lim _{u \rightarrow 1} 1-2 u-C(u, u) / 1-u
\end{aligned}
$$

where $\lambda_{l}$ and $\lambda_{u} \in[0,1]$.

\section{Definition 2. 1. 4. Elliptical Copulas}

Let $F$ be the multivariate cumulative distribution function (cdf) of an elliptical distribution. Let $F_{i}$ be CDF of the $i$ 'th margin and $F_{i}^{-1}$ be its inverse function for $i=1,2, \ldots, n$, the elliptical copula determined by $\mathrm{F}$ is;

$$
C\left(u_{1}, u_{2}, \ldots, u_{n}\right)=F\left[F_{1}^{-1}\left(u_{1}\right)+\ldots+F_{n}^{-1}\left(u_{n}\right)\right]
$$

For example; normal copulas (derived from the bivariate normal with zero mean, unitary and correlated variance) and Student's t pairs (derived from the bivariate $t$ distribution with 
zero mean, $v$ degrees of freedom and association) are two types of elliptic families.

Definition 2. 1. 5. Archimedean Copulas

An Archimedean copula is built using a generator $\phi$ as;

$$
C\left(u_{1}, u_{2}, \ldots, u_{n}\right)=\phi^{[-1]}\left[\phi_{1}\left(u_{1}\right)+\ldots+\phi_{n}\left(u_{n}\right)\right]
$$

where, $\phi^{[-1]}$ is the pseudo-inverse of the generator, $\phi$ defined by,

$$
\phi^{[-1]}=\left\{\begin{array}{cc}
\phi^{-1}(t) & 0 \leq t \leq \phi(0) \\
0 & \phi(0) \leq t \leq \phi(\infty)
\end{array}\right.
$$

In bivariate case,

$$
C\left(u_{1}, u_{2}\right)=\phi^{[-1]}\left(\phi\left(u_{1}\right)+\phi\left(u_{2}\right)\right)
$$

defines the so-called Archimedean bivariate copula function.

\section{Definition 2.1.6. Vine Copulas}

Let $T=U_{1}, U_{2}, \ldots, U_{n-1}$ denote the regular vine for $n$ variables, where $U_{i}$ is a connected tree with nodes $M_{i}=1,2, \ldots, n$ and edges $E_{i}$ for $i=2, \ldots, n-1$. In this tree structure, $U_{i}$ is a connected tree with nodes $M_{i}=E_{i-1}$ A regular vine with p variables is a vine where two edges in tree $i$ are connected by an edge in tree $i+1$, only if these edges share a common node. In general, there are totally $n(n-2) / 2$ possible edges in a regular vine for $n$ variables [19].

Definition 2.1.7. Conditional Copula

Given [19]

$f\left(x_{1}, x_{2}, \ldots, x_{n}\right)=\left(\sum_{k=2}^{n} f\left(x_{k} \mid x_{1}, x_{2}, \ldots, x_{k-1}\right)\right) f_{1}\left(x_{1}\right)$

and for distinct values of $i, j, i_{1}, \ldots, i_{m}$ with $\mathrm{i}<\mathrm{j}$ and $i_{1}<\ldots<i_{m}$ and describe

$c_{i, j \mid i_{1}, i_{2}, \ldots i_{m}}$

$=c_{i, j \mid i_{1}, i_{2}, \ldots i_{m}}\left(F\left(x_{i} \mid x_{i 1}, \ldots, x_{i m}\right), F\left(x_{j} \mid x_{i 1}, \ldots, x_{i m}\right)\right)$

where $f$ and $c$ respectively define the marginal probability density function (pdf.) and the copula density function. Then the conditional pdf can be written.;

$$
\begin{aligned}
& f\left(x_{k} \mid x_{1}, x_{2}, \ldots, x_{k-1}\right)=c_{1, k \mid 2, \ldots, k-1} f\left(x_{k} \mid x_{2}, \ldots, x_{k-1}\right) \\
& =\sum_{q=1}^{k-2}\left(c_{q, k \mid q+1, \ldots, k-1}\right) c_{k-1, k} f_{k}\left(x_{k}\right)
\end{aligned}
$$

by using equation (12) and (13) writing,

$$
f\left(x_{1}, x_{2}, \ldots, x_{n}\right)=\left(\sum_{j=1}^{n-1} \sum_{i=1}^{n-j} c_{i, i+j \mid i+1, \ldots, i+j-1}\right) \sum_{m=1}^{n} f_{m}\left(x_{m}\right) \text {. }
$$

A vine copula structure is simply defined as a nested set of trees that define binary copula functions unconditionally in the first tree and conditionally for the rest of the related trees. This structure is as follows;

\section{Definition 2.1.8.C Vine Copula}

It is a sort of regular vine distribution where each tree has a unique node connected to all the other nodes in the tree. It only uses star shaped trees and is useful for sorting by importance. The corresponding probability density function (pdf) can be written as:

$$
\begin{aligned}
& f\left(x_{1}, x_{2}, \ldots, x_{n}\right)=\prod_{k=1}^{n} f\left(x_{k}\right) \prod_{j=1}^{n-1} \prod_{i=1}^{n-j} c_{j, j+i \mid 1, \ldots, j-1} \\
& \left\{F\left(x_{j} \mid x_{1}, \ldots, x_{j-1}\right), F\left(x_{j+i} \mid x_{1}, \ldots, x_{j-1}\right)\right\} .
\end{aligned}
$$

\section{Definition 2.1.8.D Vine Copula}

$\mathrm{D}$-vine is another special case for the normal vine tree structure which has no nodes in any tree but connects to more than two edges. It uses paths like trees and is useful for the temporal ordering of variables. The density function (pdf) can be written:

$$
\begin{aligned}
& f\left(x_{1}, x_{2}, \ldots, x_{n}\right)=\prod_{k=1}^{n} f\left(x_{k}\right) \prod_{j=1}^{n-1} \prod_{i=1}^{n-j} c_{i, i+j \mid i+1, \ldots, i+j-1} \\
& \left\{F\left(x_{i} \mid x_{i+1}, \ldots, x_{i+j-1}\right) F\left(x_{i+j} \mid x_{i+1}, \ldots, x_{i+j-1}\right)\right\} .
\end{aligned}
$$

\section{Results}

We use magnitude and depth of Eastern Anatolian fault line and North Anatolian fault line data of stations the period 19992019. Table 1 and Table 2 summarizes statistics of magnitude and depth of Eastern Anatolian fault line and North Anatolian fault line data. In Table 1, Table 2 and Figure 1, Figure 2 shows different mean values for the for data set, and the corresponding standard deviations are different. Skewness of data set is positive, indicating that this data is skewed right. The high kurtosis of data set reveals that extreme value changes often occur when the tail of return distributions shows fatness. The Jarque-Bera (JB) test shows that the normality of each return series distribution is strongly rejected at 0.05 level, which means all price index distributions are non-normal. The empirical distribution functions used in modelling the dependence of Eastern Anatolian fault line and North Anatolian fault line depth and magnitude data pairs are as shown in Figure 3,4,5,6,7,8,9,10 and Table 3,4. For this, we used C Vine and D Vine copula. In table 3,4,5 and Figure 1, 2, 3, 4 it is shown that for the relationship between North Anatolian fault line depth and North Anatolian fault line magnitude, in table 3,4,5 Figure5,6,7,8 it is shown that for the relationship between Eastern Anatolian fault line depth and Eastern Anatolian fault line magnitude, we use C Vine and D Vine copula modelling. In Table 3,4, the preferred copula families for branching and the parameter summaries of these families are given. In Figure figure $3,4,5,6,7,8,9,10$ for C Vine, D Vine matrix summaries and 
appropriate branch graphs are shown. From table 3,4,5 Figure $3,4,5,6,7,8,9,10$ for dependency structure of North Anatolian fault line depth, $\mathrm{C}$ vine branching was found to be appropriate according to the information criteria. Similarly, for North Anatolian fault line magnitude data. From table 3,4,5 Figure $3,4,5,6,7,8,9,10$, for dependency structure of North Anatolian fault line magnitude, $\mathrm{C}$ vine branching was found to be appropriate according to the information criteria, for Eastern Anatolian fault line depth data, in table 3,4,5 Figure $3,4,5,6,7,8,9,10$, for dependency structure of Eastern Anatolian fault line depth, D vine branching was found to be appropriate according to the information criteria and for Eastern Anatolian fault line magnitude data, in table 3,4,5 Figure 3,4,5,6,7,8,9,10 for dependency structure of Eastern Anatolian fault line magnitude, D vine branching was found to be appropriate according to the information criteria.

Table 1. Summary Statistics for Eastern Anatolian fault line

\begin{tabular}{lccccc} 
& Adiyaman & Elazı g & K.Maraş & Malatya & Şırnak \\
\cline { 2 - 6 } Depth & & & & & \\
Mean & 8,243636 & 16,15455 & 18,00909 & 17,65455 & 15,19273 \\
Maksimum & 15,35000 & 45,40000 & 64,20000 & 45,40000 & 35,00000 \\
Minumum & 2,600000 & 2,000000 & 6,900000 & 9,500000 & 5,000000 \\
Std.Dev & 3,393636 & 15,43421 & 16,86265 & 11,13008 & 10,19934 \\
Skewness & 0,344330 & 0,929670 & 2,087951 & 1,594602 & 1,348772 \\
Kurtosis & 3,165211 & 2,248423 & 6,270340 & 4,557203 & 3,287966 \\
Jarqure Bera & 0,229876 & 1,843424 & 12,89442 & 5,773121 & 3,373180 \\
Probability & 0,891421 & 0,397837 & 0,001585 & 0,055768 & 0,185150 \\
Magnitude & & & & & \\
Mean & 4,500000 & 4,309091 & 4,145455 & 4,318182 & 4,300000 \\
Maksimum & 5,500000 & 5,100000 & 4,500000 & 5,100000 & 5,100000 \\
Minumum & 4,000000 & 4,000000 & 4,000000 & 4,000000 & 4,000000 \\
Std.Dev & 0,473286 & 0,311302 & 0,180907 & 0,345885 & 0,354965 \\
Skewness & 0,908170 & 1,601171 & 0,874018 & 1,192958 & 1,097454 \\
Kurtosis & 2,771923 & 4,845286 & 2,344167 & 3,250882 & 3,163643 \\
Jarqure Bera & 1,535924 & 6,260866 & 1,597635 & 2,637954 & 2,220351 \\
Probability & 0,463958 & 0,043699 & 0,449861 & 0,267409 & 0,329501
\end{tabular}

Table 2. Summary Statistics for North Anatolian fault line

\begin{tabular}{lccccc} 
& Çankırı & Düzce & Erzincan & Izmit & Sakarya \\
\cline { 2 - 6 } Depth & & & & & \\
Mean & 11,04286 & 20,00000 & 27,87143 & 13,51429 & 17,64286 \\
Maksimum & 22,30000 & 65,60000 & 66,10000 & 22,60000 & 56,00000 \\
Minumum & 7,000000 & 10,00000 & 7,000000 & 7,000000 & 3,700000 \\
Std.Dev & 5,110074 & 20,70394 & 23,37404 & 5,863852 & 17,79671 \\
Skewness & 1,791256 & 1,828218 & 0,512661 & 0,553095 & 1,668841 \\
Kurtosis & 4,681279 & 4,624956 & 1,852352 & 1,783947 & 4,292637 \\
Jarqure & 4,567817 & 4,669584 & 0,690778 & 0,788212 & 3,736549 \\
$\begin{array}{l}\text { Bera } \\
\text { Probability }\end{array}$ & 0,101885 & 0,096831 & 0,707945 & 0,674283 & 0,154390 \\
Magnitude & 4,042857 & 4,685714 & 5,200000 & 4,928517 & 4,300000 \\
Mean & 4,200000 & 7,100000 & 6,600000 & 7,600000 & 4,500000 \\
Maksimum & 4,000000 & 4,000000 & 4,600000 & 4,000000 & 4,100000 \\
Minumum & & & & & \\
Std.Dev & 0,078680 & 1,080785 & 0,797914 & 1,237894 & 0,163299 \\
Skewness & 1,357727 & 1,916392 & 0,822842 & 1,652398 & 0,248039 \\
Kurtosis & 3,233728 & 4,893136 & 2,175256 & 4,331803 & 1,421875 \\
Jarqure & 2,166593 & 5,329972 & 0,988305 & 3,702817 & 0,798167 \\
Bera & & & & & \\
Probability & 0,338478 & 0,069600 & 0,610088 & 0,157016 & 0,670935 \\
& & & & &
\end{tabular}
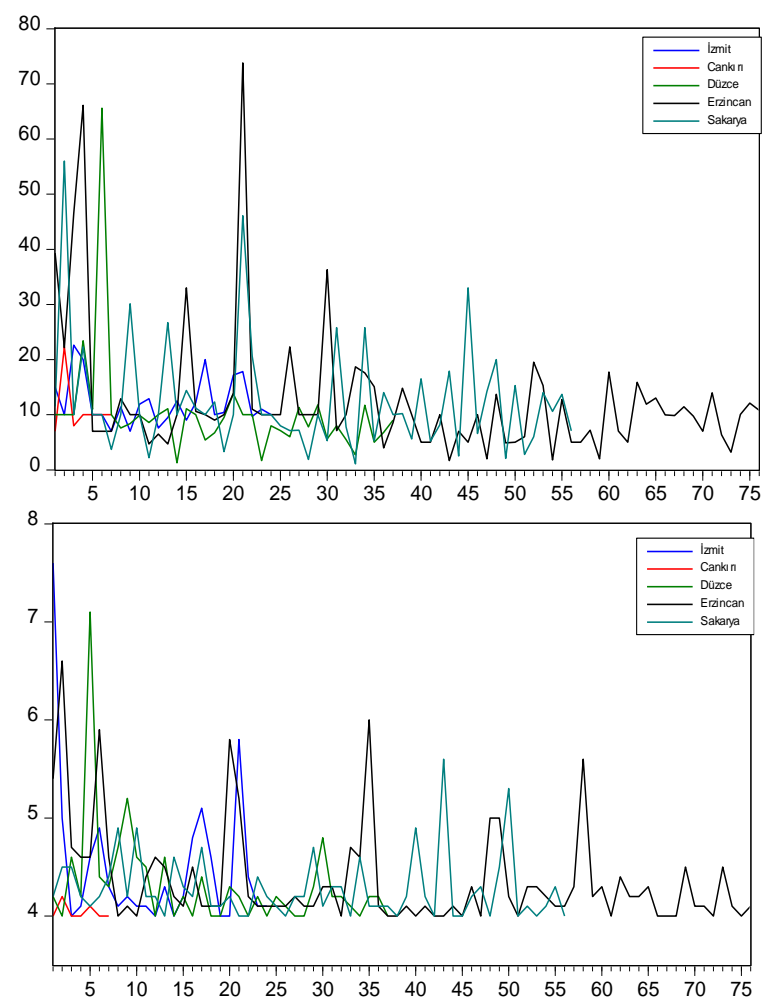

Figure 1. Respectively, Depth and Magnitude of North Anatolian fault line.
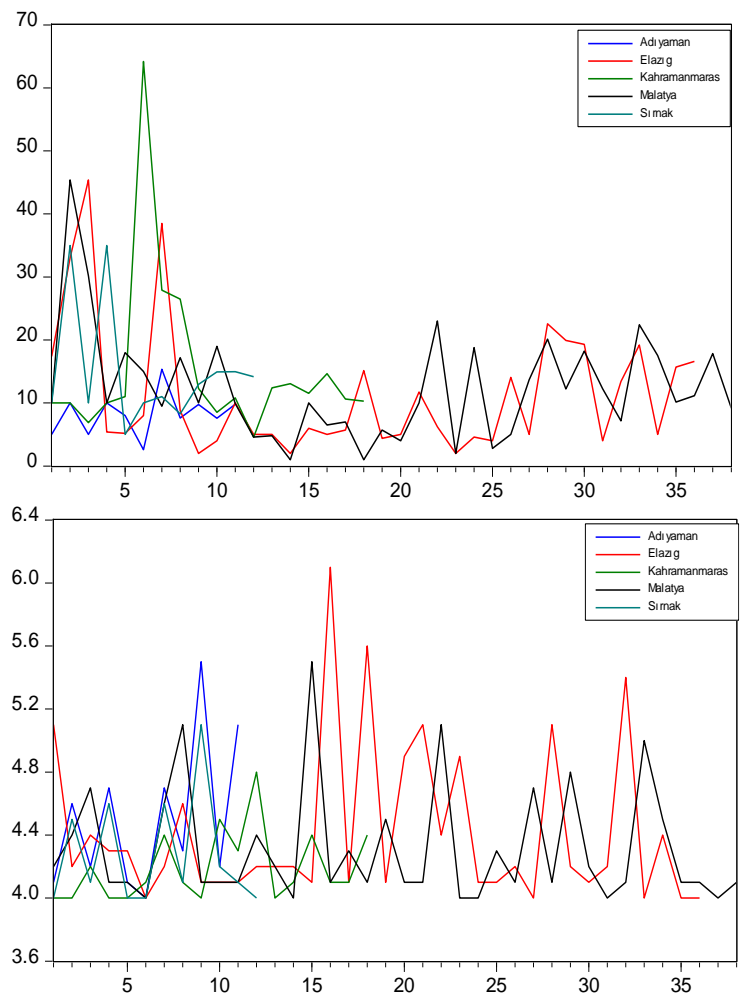

Figure 2. Respectively, Depth and Magnitude of Eastern Anatolian fault 
Table 3. C-vine copula estimation results.

\begin{tabular}{|c|c|c|c|c|c|c|}
\hline Depth of North Anatolian fault line & Copula & Par1 & Par2 & Tau & Lower tail dependency & Upper tail dependency \\
\hline$C_{\text {Çanklrt-İzmit }}$ & I & - & - & 0.00 & - & - \\
\hline$C_{\text {Düzce-İzmit }}$ & $\mathrm{C}$ & 2.88 & 0.00 & 0.59 & - & 0.79 \\
\hline$C_{\text {Sakarya-İmit }}$ & $\mathrm{C}$ & 1.05 & 0.00 & 0.34 & - & 0.52 \\
\hline$C_{\text {Erzincan-İzmit }}$ & $\mathrm{C}$ & 0.56 & 0.00 & 0.22 & - & 0.29 \\
\hline$C_{\text {Erzincan,Çankirılizzmit }}$ & I & - & - & 0.00 & - & - \\
\hline$C_{\text {Erzincan,Düzcel İzmit }}$ & Tawn & 18.00 & 0.90 & 0.85 & 0.89 & - \\
\hline$C_{\text {Erzincan,Sakarya/İzmit }}$ & Tawn2_180 & 16.10 & 0.90 & 0.85 & - & 0.89 \\
\hline$C_{\text {Sakarya,Çankırı,Erzincan/İzmit }}$ & I & - & - & 0.00 & - & - \\
\hline$C_{\text {Sakarya,Dïzce,Erzincan/Izmit }}$ & SBB6 & 6.00 & 6.00 & 0.95 & - & 0.98 \\
\hline$C_{\text {Düzce,ÇankirlSakarya,Erzincan/İzmit }}$ & I & - & - & 0.00 & - & - \\
\hline Magnitude of North Anatolian fault line & Copula & Par1 & Par2 & Tau & Lower tail dependency & Upper tail dependency \\
\hline$C_{\text {Çankirl-İzmit }}$ & I & - & - & 0.00 & - & - \\
\hline$C_{\text {Düzce-Izmit }}$ & $\mathrm{C}$ & 2.88 & 0.00 & 0.59 & - & 0.79 \\
\hline$C_{\text {Sakarya-Izmit }}$ & $\mathrm{N}$ & 0.03 & 0.00 & 0.02 & - & - \\
\hline$C_{\text {Erzincan-İzmit }}$ & $\mathrm{C}$ & 0.56 & 0.00 & 0.22 & - & 0.29 \\
\hline$C_{\text {Erzincan,Çankirl/İzmit }}$ & I & - & - & 0.00 & - & - \\
\hline$C_{\text {Erzincan,Dïzcel İzmit }}$ & Tawn & 18.00 & 0.90 & 0.85 & 0.89 & - \\
\hline$C_{\text {Erzincan,Sakarya/Izmit }}$ & $\mathrm{J}$ & 8.48 & 0.00 & 0.79 & 0.91 & - \\
\hline$C_{\text {Sakarya,Çankırı/Erzincan İzmit }}$ & I & - & - & 0.00 & - & - \\
\hline$C_{\text {Sakarya,Düzce/Erzincan İzmit }}$ & $\mathrm{C} 90$ & -0.96 & 0.00 & -0.32 & - & - \\
\hline$C_{\text {Düzce,Çankirl/Sakarya, Erzincan İzmit }}$ & I & - & - & 0.00 & - & - \\
\hline Depth of Eastern Anatolian fault line & Copula & Par1 & Par2 & Tau & Lower tail dependency & Upper tail dependency \\
\hline$C_{\text {Elazı̆̆-Şırnak }}$ & Tawn2_90 & -5.32 & 0.06 & -0.06 & - & - \\
\hline$C_{\text {Malatya-Şirnak }}$ & Tawn90 & -20.00 & 0.30 & -0.29 & - & - \\
\hline$C_{\text {Adiyaman-Sirnak }}$ & Tawn2 & 5.45 & 0.47 & 0.42 & 0.46 & - \\
\hline$C_{\text {Kahramanmaraş-Şırnak }}$ & Tawn2_270 & -18.59 & 0.10 & -0.10 & - & - \\
\hline$C_{\text {Kahramanmaraş,Elazı̆g/Şırnak }}$ & Tawn2_270 & -20.00 & 0.06 & -0.06 & - & - \\
\hline$C_{\text {Kahramanmaraş,Malatya/Şırnak }}$ & J90 & -1.24 & 0.00 & -0.12 & - & - \\
\hline$C_{\text {Kahramanmaraş,Adlyaman/Şırnak }}$ & Tawn & 4.17 & 0.43 & 0.38 & 0.43 & - \\
\hline$C_{\text {Adıyaman,Elazığ,Kahramanmaraş/Şırnak }}$ & SBB1 & 0.93 & 1.00 & 0.32 & 0.48 & 0.00 \\
\hline$C_{\text {Adıyaman,Malatya,Kahramanmaraș/Şırnak }}$ & Tawn90 & -20.00 & 0.20 & -0.20 & - & - \\
\hline$C_{\text {Malatya,Elazığ,Adıyaman,Kahramanmaraş/Şırnak }}$ & $\mathrm{t}$ & 0.07 & 2.00 & 0.04 & 0.20 & 0.20 \\
\hline
\end{tabular}




\begin{tabular}{|c|c|c|c|c|c|c|}
\hline Magnitude of Eastern Anatolian fault line & Copula & Par1 & Par2 & Tau & Lower tail dependency & Upper tail dependency \\
\hline$C_{\text {Elazığ-Şırnak }}$ & $\mathrm{t}$ & 0.03 & 30.00 & 0.02 & 0.00 & 0.00 \\
\hline$C_{\text {Malatya-Ssirnak }}$ & $\mathrm{J} 270$ & -1.07 & 0.00 & -0.04 & - & - \\
\hline$C_{\text {Adlyaman-Sirnak }}$ & SJ & 15.94 & 0.00 & 0.88 & - & 0.96 \\
\hline$C_{\text {Kahramanmaraş-Şırnak }}$ & SJ & 15.94 & 0.00 & 0.88 & - & 0.96 \\
\hline$C_{\text {Kahramanmaraş,Elazı̆̆/Şırnak }}$ & $\mathrm{C} 270$ & -0.00 & 0.00 & -0.00 & - & - \\
\hline$C_{\text {Kahramanmaraş,Malatya/Şırnak }}$ & $\mathrm{F}$ & -0.88 & 0.00 & -0.10 & - & - \\
\hline$C_{\text {Kahramanmaraş,Adiyaman/Şırnak }}$ & BB7_270 & -1.02 & -0.00 & -0.01 & - & - \\
\hline$C_{\text {Adıyaman,Elazığ,Kahramanmaraş/Şırnak }}$ & Tawn & 2.76 & 0.32 & 0.26 & 0.30 & - \\
\hline$C_{\text {Adıyaman,Malatya,Kahramanmaraş/Şırnak }}$ & Tawn2_180 & 1.85 & 0.00 & 0.00 & - & 0.00 \\
\hline$C_{\text {Malatya,Elazığ,Adıyaman,Kahramanmaraş/Şırnak }}$ & G & 1.09 & 0.00 & 0.08 & 0.11 & - \\
\hline
\end{tabular}

Table 4. D-vine copula estimation results.

Depth of North Anatolian fault line

\begin{tabular}{|c|c|c|c|c|c|c|}
\hline Depth of North Anatolian fault line & Copula & Par1 & Par2 & Tau & Lower tail dependency & Upper tail dependency \\
\hline$C_{\text {Erzincan-Sakarya }}$ & $\mathrm{C}$ & 4.32 & 0.00 & 0.68 & - & 0.85 \\
\hline$C_{\text {Sakarya-İmir }}$ & $\mathrm{C}$ & 1.05 & 0.00 & 0.34 & - & 0.52 \\
\hline$C_{\dot{I}_{\text {zmir-Düzce }}}$ & $\mathrm{C}$ & 2.88 & 0.00 & 0.59 & - & 0.79 \\
\hline$C_{\text {Düzce-Çankırı }}$ & I & - & - & 0.00 & - & - \\
\hline$C_{\dot{I z m i t}, \text { Erzincan/Sakarya }}$ & $\mathrm{N}$ & -0.78 & 0.00 & -0.57 & - & - \\
\hline$C_{\text {Düzce,İzmit/Sakarya }}$ & Tawn2 & 20.00 & 0.93 & 0.89 & 0.92 & - \\
\hline$C_{\text {Çankırl, İzmit/Düzce }}$ & I & - & - & 0.00 & - & - \\
\hline$C_{\text {Düzce,Erzincan/Sakarya,İzmir }}$ & BB7_270 & -1.00 & -1.65 & -0.45 & - & - \\
\hline$C_{\text {Çankırı,Sakarya/Düzce, İzmir }}$ & I & - & - & 0.00 & - & - \\
\hline$C_{\text {Çanklr }, \text { Erzincan/Düzce, İmir }, \text { Sakarya }}$ & I & - & - & 0.00 & - & - \\
\hline Magnitude of North Anatolian fault line & Copula & Par1 & Par2 & Tau & Lower tail dependency & Upper tail dependency \\
\hline$C_{\text {Erzincan-İzmir }}$ & $\mathrm{C}$ & 0.56 & 0.00 & 0.22 & - & 0.29 \\
\hline$C_{\text {Izmir-Sakarya }}$ & $\mathrm{N}$ & 0.03 & 0.00 & 0.02 & - & - \\
\hline$C_{\text {Sakarya-Dïzce }}$ & $\mathrm{C}$ & 0.02 & 0.00 & 0.01 & - & 0.00 \\
\hline$C_{\text {Düzce-Çankırı }}$ & I & - & - & 0.00 & - & - \\
\hline$C_{\text {Sakarya,İzmit/Erzincan }}$ & $\mathrm{J}$ & 8.48 & 0.00 & 0.79 & 0.91 & - \\
\hline$C_{\text {Düzce, Izmit/Sakarya }}$ & $\mathrm{C}$ & 3.57 & 0.00 & 0.64 & - & 0.82 \\
\hline$C_{\text {Çankırl,Sakarya/Düzce }}$ & I & - & - & 0.00 & - & - \\
\hline$C_{\text {Düzce,Erzincan/Sakarya, Izmit }}$ & Tawn90 & -10.77 & 0.31 & -0.30 & - & - \\
\hline$C_{\text {Çankırı, İzmit/Düzce,Sakarya }}$ & I & - & - & 0.00 & - & - \\
\hline$C_{\text {Çanklr }, \text { Erzincan/Düzce,Sakarya, İzmit }}$ & I & - & - & 0.00 & - & - \\
\hline
\end{tabular}


Bitlis Eren University Journal of Science and Technology 11 (2) (2021) 60-75

\begin{tabular}{|c|c|c|c|c|c|c|}
\hline Depth of Eastern Anatolian fault line & Copula & Par1 & Par2 & Tau & Lower tail dependency & Upper tail dependency \\
\hline$C_{\text {Şırnak-Kahramanmaraș }}$ & Tawn90 & -18.59 & 0.10 & -0.10 & - & - \\
\hline$C_{\text {Kahramanmaraş-Adiyaman }}$ & $\mathrm{t}$ & 0.22 & 2.00 & 0.14 & 0.26 & 0.26 \\
\hline$C_{\text {Adiyaman-Malatya }}$ & $\mathrm{C} 270$ & -0.83 & 0.00 & -0.29 & - & - \\
\hline$C_{\text {Malatya-Elazı̆ }}$ & $\mathrm{t}$ & 0.48 & 2.00 & 0.32 & 0.38 & 0.38 \\
\hline$C_{\text {Adlyaman,Şırnak/Kahramanmaraş }}$ & $\mathrm{J}$ & 2.88 & 0.00 & 0.50 & 0.73 & - \\
\hline$C_{\text {Malatya }, \text { Kahramanmaraş,/Adiyaman }}$ & $\mathrm{C} 90$ & -0.70 & 0.00 & -0.26 & - & - \\
\hline$C_{\text {Elazığ,Adiyaman/Malatya }}$ & $\mathrm{J}$ & 1.20 & 0.00 & 0.10 & 0.21 & - \\
\hline$C_{\text {Malatya,Şırnak/Adıyaman,Kahramanmaraş }}$ & Tawn2_270 & -19.27 & 0.43 & -0.42 & - & - \\
\hline$C_{\text {Elazığ,Kahramanmaraş/Malatya,Adlyaman }}$ & Tawn & 12.92 & 0.09 & 0.09 & 0.09 & - \\
\hline$C_{\text {Elazı̆̆,Şırnak/Malatya,Adıyaman,Kahramanmaraş }}$ & BB1_90 & -1.13 & -1.00 & -0.36 & - & - \\
\hline Magnitude of Eastern Anatolian fault line & Copula & Par1 & Par2 & Tau & Lower tail dependency & Upper tail dependency \\
\hline$C_{\text {Adlyaman-Şirnak }}$ & SJ & 15.94 & 0.00 & 0.88 & - & 0.96 \\
\hline$C_{\text {Şırnak-Kahramanmaraș }}$ & SJ & 15.94 & 0.00 & 0.88 & - & 0.96 \\
\hline$C_{\text {Kahramanmarass-Malatya }}$ & SC & 0.13 & 0.00 & 0.06 & 0.00 & - \\
\hline$C_{\text {Malatya-Elazı̆̆ }}$ & Tawn270 & -5.29 & 0.12 & -0.11 & - & - \\
\hline$C_{\text {Kahramanmaraş,Adıyaman/Şırnak }}$ & BB7_270 & -1.02 & -0.00 & -0.01 & - & - \\
\hline$C_{\text {Malatya,Şırnak/Kahramanmaraş }}$ & $\mathrm{C}$ & 0.00 & 0.00 & 0.00 & - & 0.00 \\
\hline$C_{\text {Elazığ,Kahramanmaraş/Malatya }}$ & SJ & 1.01 & 0.00 & 0.00 & - & 0.01 \\
\hline$C_{\text {Malatya,Adıyaman/Şırnak,Kahramanmaraş }}$ & $\mathrm{N}$ & 0.06 & 0.00 & 0.04 & - & - \\
\hline$C_{\text {Elazığ,Şırnak/Kahramanmaraş,Malatya }}$ & Tawn2_180 & 20.00 & 0.01 & 0.00 & - & 0.01 \\
\hline$C_{\text {Elazığ,Adıyaman/Malatya,Kahramanmaraş,Şırnak }}$ & $\mathrm{N}$ & 0.16 & 0.00 & 0.10 & - & - \\
\hline
\end{tabular}

Table 5. Comparison of the C-vine and D-vine.

\begin{tabular}{ccc}
\multicolumn{4}{c}{ Table 5. Comparison of the C-vine and D-vine. } \\
\hline Tree & C vine & D vine. \\
\hline Depth of North Anatolian fault line & & \\
\hline Loglike & 62.7 & 55.79 \\
AIC & -107.39576 & -95.57715 \\
BIC & -86.42 & -76.93 \\
\hline Magnitude of North Anatolian fault line & & \\
\hline Loglike & 38.83 & 39.39 \\
AIC & -63.66569 & -60.78 \\
BIC & -47.35 & -44.47 \\
\hline Depth of Anatolian fault line & & \\
\hline Loglike & 21.47 & 22.52 \\
AIC & -10.93 & -13.034703 \\
BIC & 20.18 & 13.17 \\
\hline Magnitude of Anatolian fault line & \\
\hline Loglike & 30267.22 & 30285.76 \\
AIC & -60506.43 & -60545.53 \\
BIC & -60483.51 & -60524.24 \\
\hline
\end{tabular}


Tree 1

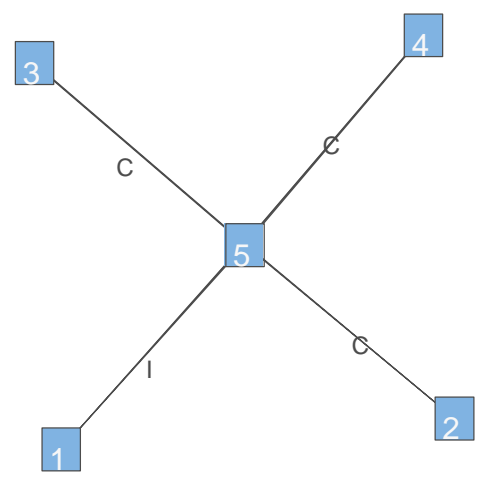

Tree 3

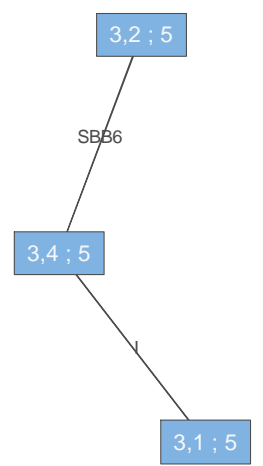

Tree 2

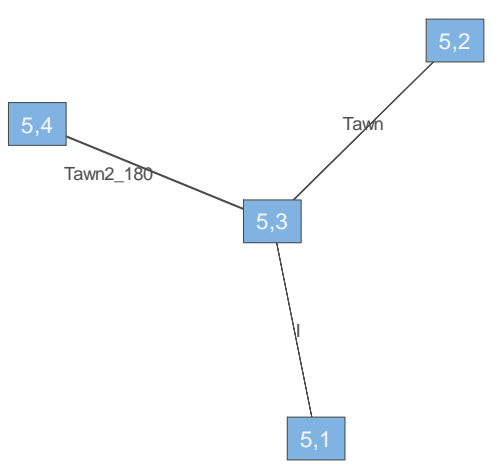

Tree 4
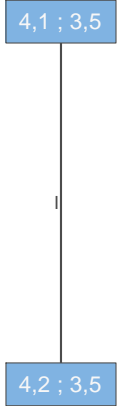

Figure 3. C Vine Copula Summary for Depth of North Anatolian fault line (1. Cankırı, 2. Düzce, 3. Erzincan, 4. Sakarya, 5. İzmit) 
Tree 1

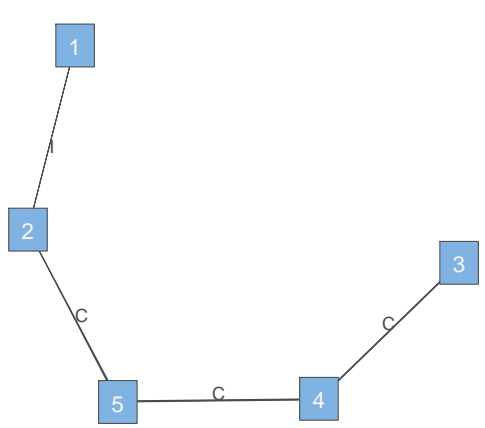

Tree 3

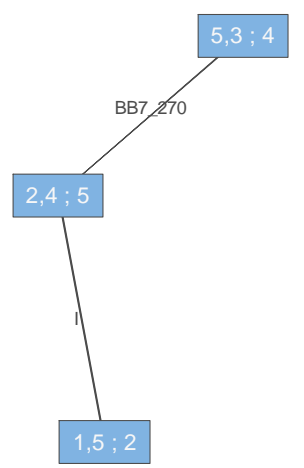

Tree 2

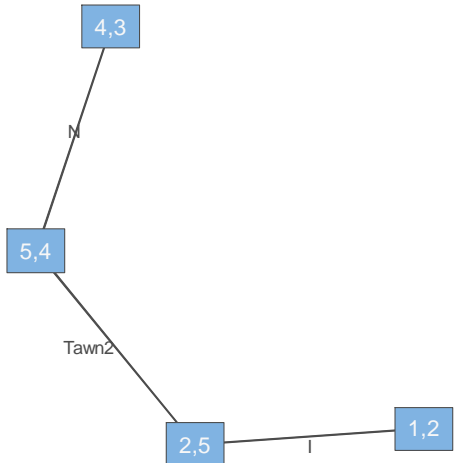

Tree 4

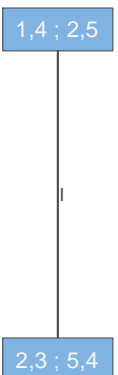

Figure 4. D Vine Copula Summary for Depth of North Anatolian fault line (1. Cankırı, 2. Düzce, 3. Erzincan, 4. Sakarya, 5. İzmit). 


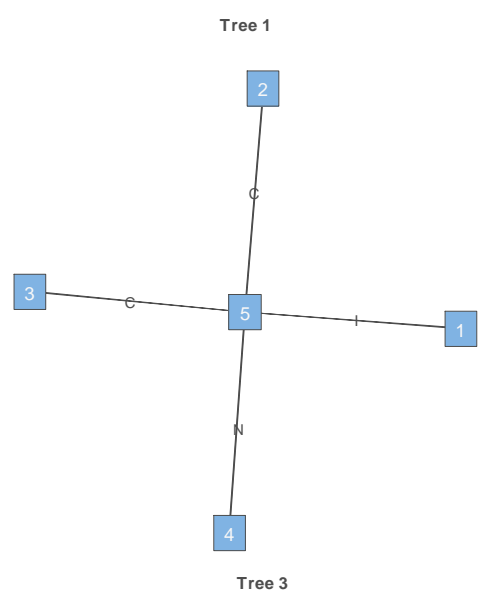

Tree 3

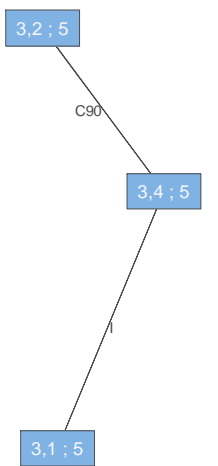

Tree 2

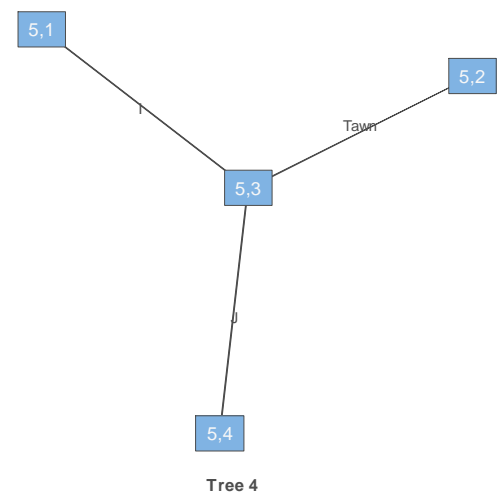

4,$1 ; 3,5$

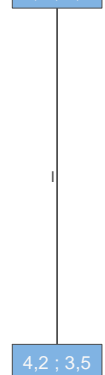

Figure 5. C Vine Copula Summary for magnitude of North Anatolian fault line (1. Cankırı, 2. Düzce, 3. Erzincan, 4. Sakarya, 5. İzmit). 
Tree 1

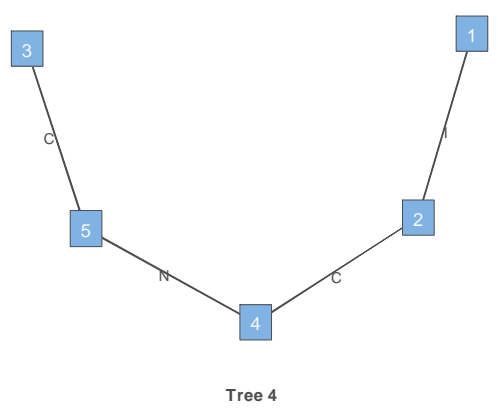

1,$5 ; 2,4$

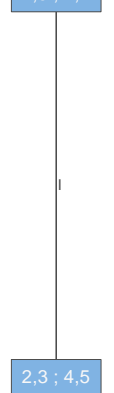

Tree 2
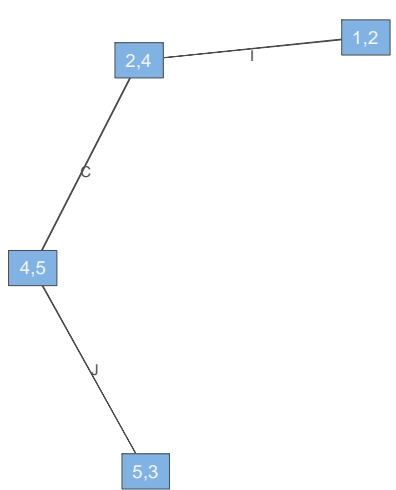

Tree 3

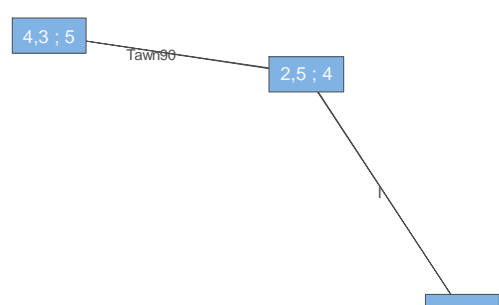

Figure 6. D Vine Copula Summary for Magnitude of North Anatolian fault line (1. Cankırı, 2. Düzce, 3 .Erzincan, 4. Sakarya, 5. İzmit). 
Tree 1
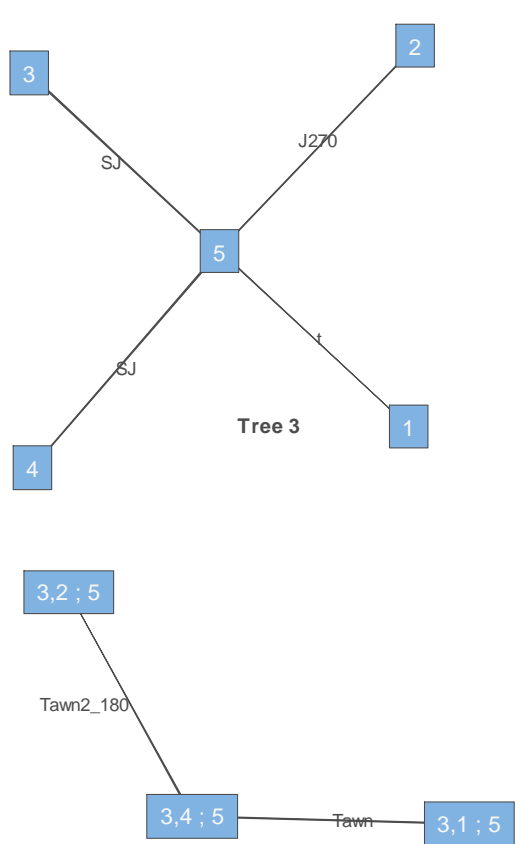

Tree 2

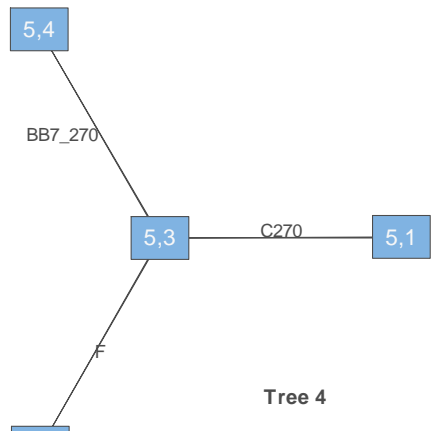

5,2

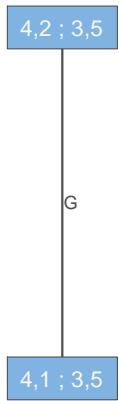

Figure 7. C Vine Copula Summary for depth of Eastern Anatolian fault line (1. Elazıg, 2. Malatya, 3. Kahramanmaras, 4. Adıyaman, 5. Sirnak) 
Tree 1
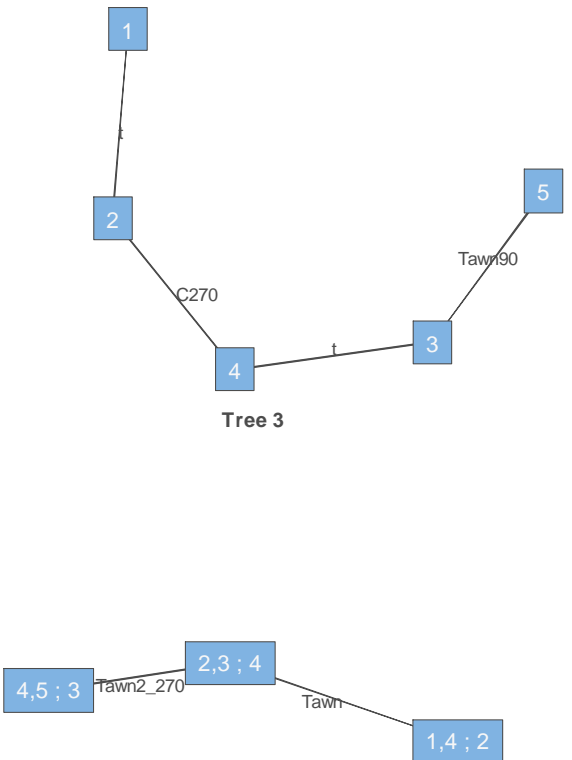

Tree 2

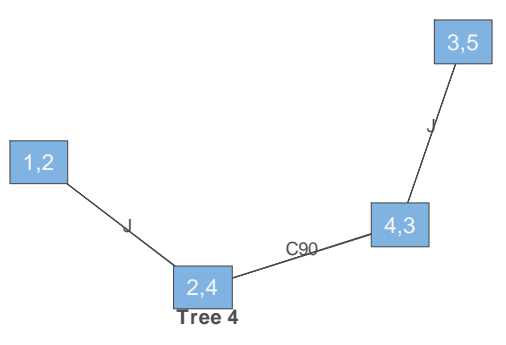

\section{2,$5 ; 4,3$}

BB1_90

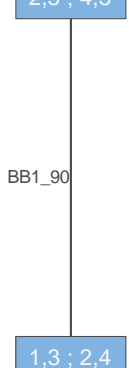

Figure 8. D Vine Copula Summary for depth of Eastern Anatolian fault line (1. Elazıg, 2. Malatya, 3. Kahramanmaras, 4. Adıyaman, 5. Sirnak). 

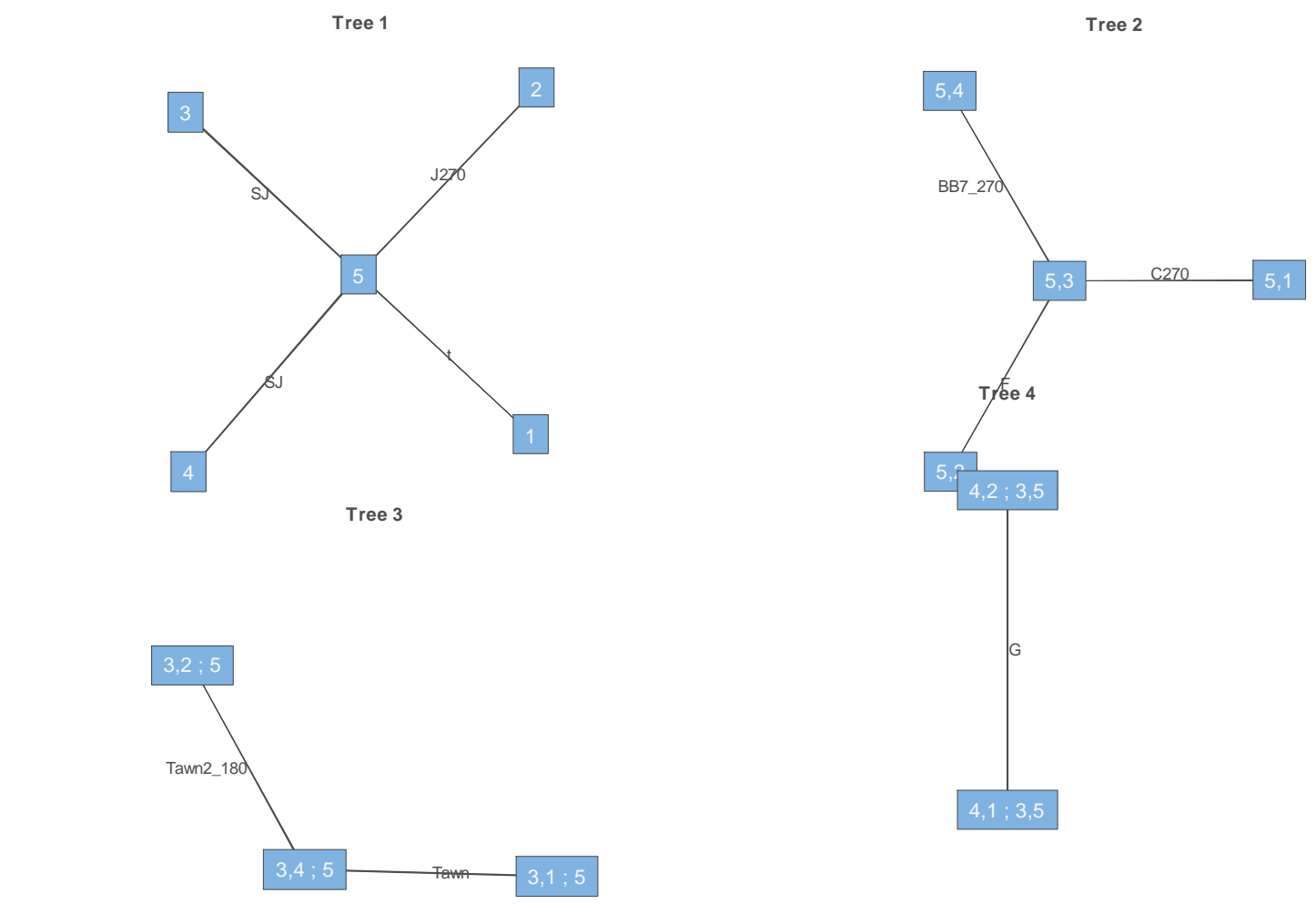

Figure 9. C Vine Copula Summary for Magnitude of Eastern Anatolian fault line (1. Elazıg, 2. Malatya, 3. Kahramanmaras, 4. Adıyaman, 5. Sirnak) 
Tree 1

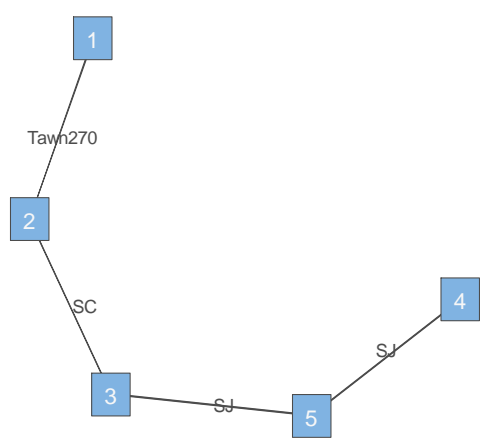

Tree 3

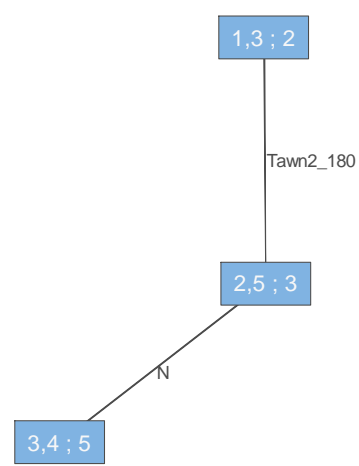

Tree 2

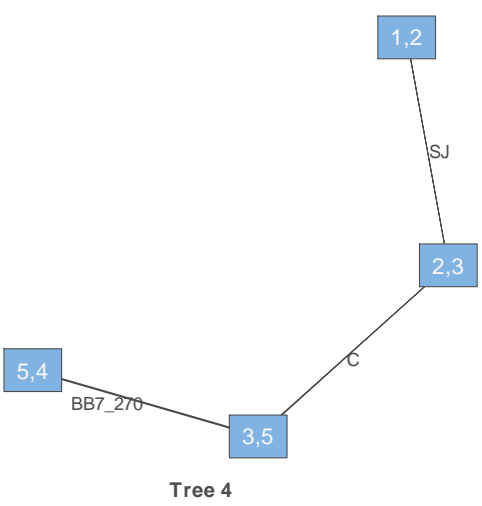

Tree 4

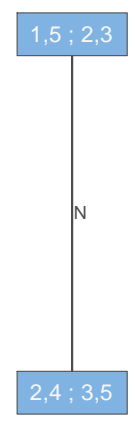

Figure 10. D Vine Copula Summary for Magnitude of Eastern Anatolian fault line (1. Elazıg, 2. Malatya, 3. Kahramanmaras, 4. Adıyaman, 5. Sirnak)

\section{Acknowledgments}

Thank you to the Kandilli Observatory and Earthquake Research Institute and Turkey's Interior Ministry Disaster and Emergency Management Presidency for our data set.

\section{References}

[1] Sriboonchitta, S., \& Chaiboonsri, C. 2013. The dynamics Comovement toward among capital markets in ASEAN exchanges: CD Vine Copula approach. Procedia Economics and Finance, 5, 696-702.

[2] Chaiboonsri, C., \& Singvejsakul, J. 2017. The Dynamics Co- 
Movement toward and the Pattern of Relation among Stock Market in World Exchanges during the Period 2000 2016: CD vine Copula Approach. International Journal of Intelligent Technologies \& Applied Statistics, 10(2).

[3] Hikmah, I. R., Saefuddin, A., \& Mangku, I. 2017. Identification of Dependent Structure and Prediction of Composite Stock Price Index with CD Vine Copula Approach. International Journal of Scientific \& Engineering Research, 7, 249-252.

[4] Schepsmeier, U., \& Brechmann, E. C. 2013. Modeling dependence with $\mathrm{C}$-and $\mathrm{D}$-vine copulas: The R package CD vine. J. Stat. Software, 52(3), 1-27.

[5] Chokethaworn, K., Chaitip, P., Sriwichailamphan, T., \& Chaiboonsri, C. 2013. The Dependence Structure and Comovement toward between Thai's Currency and Malaysian's Currency: Markov Switching Model in Dynamic Copula Approach (MSDC). Procedia Economics and Finance, 5, 152-161.

[6] Evkaya, Ö. 0. 2018. Mixture of vines for dependence modeling: Finite mixture and CD-vine approaches with applications.

[7] Karakas, A., Demir, A., Çalik, S. 2019. Interdependence of Bitcoin and Other Crypto Money Indicators: Cd Vine Copula Approach. Bitlis Eren Üniversitesi Fen Bilimleri Dergisi, 9(4), 1527-1536.

[8] Kim, D., Kim, J.-M., Liao, S.-M., and Jung, Y.-S., 2013. Mixture of $d$-vine copulas for modeling dependence. Computational Statistics and Data Analysis, 64(C):1-19.

[9] Yan, J. 2007. Enjoy the joy of copulas: with a package copula. Journal of Statistical Software, 21(4), 1-21.

[10] Pourkhanali, A., Kim, J. M., Tafakori, L., Fard, F. A. (2016). Measuring systemic risk using vine copula. Economic modelling, 53, 63-74.

[11] Nguyen-Huy, T., Deo, R. C., Mushtaq, S., An-Vo, D. A., \& Khan, S. 2018. Modeling the joint influence of multiple synoptic-scale, climate mode indices on Australian wheat yield using a vine copula-based approach. European journal of agronomy, 98, 65-81.

[12] İnç, M., Korpinar, Z. S., Al Qurashi, M. M., \& Baleanu, D. 2016. A new method for approximate solutions of some nonlinear equations: Residual power series method.Advances in Mechanical Engineering, 8(4), 1687814016644580.

[13] Acay, B., Inc, M. 2020. Fractional modeling of temperature dynamics of a building with singular kernels. Chaos, Solitons \& Fractals, 110482.

[14] Houwe, A., Inc, M., Doka, S. Y., Acay, B., \& Hoan, L. V. C. 2020. The discrete tanh method for solving the nonlinear differential-difference equations. International Journal of Modern Physics B, 34(19), 2050177.

[15] Akinlar, M. A., Inc, M., Gómez-Aguilar, J. F., \& Boutarfa, B. 2020. Solutions of a disease model with fractional white noise. Chaos, Solitons \& Fractals, 109840.

[16] Akinlar, M. A., Tchier, F., Inc, M. 2020. Chaos control and solutions of fractional-order Malkus waterwheel model. Chaos, Solitons \& Fractals, 135, 109746.

[17] Korpinar, Z., Tchier, F., Inc, M., Bousbahi, F. T., Tawfiq, F. M., \& Akinlar, M. A. 2020. Applicability of time conformable derivative to Wick-fractional-stochastic PDEs. Alexandria Engineering Journal.

[18] Hashemi, M. S., Inc, M., Yusuf, A. 2020. On threedimensional variable order time fractional chaotic system with nonsingular kernel. Chaos, Solitons \& Fractals, 133, 109628.

[19] Dorota K., and Roger, C., 2006, Uncertainty Analysis with High Dimensional Dependence Modelling. John Wiley and Sons, Ltd. 